\title{
Location Lookup Framework using Seamless LBS Technology
}

\author{
Nanju Kim \\ Department of Computer Engineering \\ Hannam University \\ Daejeon, Republic of Korea \\ 91knj@naver.com
}

\author{
Euiin Choi* \\ Department of Computer Engineering \\ Hannam University \\ Daejeon, Republic of Korea \\ eichoi@hnu.kr
}

\begin{abstract}
Recent various crimes and lost stuff and the like are recognized as a social problem. And users may be the subject of such crimes. To address this, the demand about location information transmission is a situation that is increasing. Therefore various institutions are providing location information service using a mobile device. But, Mobile has provided the location service using the GPS. So it is low accuracy. In this paper, we investigated for Seamless LBS technology that accuracy is higher than accuracy of GPS in mobile environment. And, we proposed a new location lookup framework that complements traditional location lookup techniques.
\end{abstract}

Keywords- Seamless LBS, Location information, Location lookup technique.

\section{INTRODUCTION}

Users have been readily exposed with respect to the recent various crimes. So it has emerged as a social issue. Accordingly, the effort is continuing for provide the user location information. So, there is increasing interest in and a need for techniques for providing accurate location. LBS (Location Based Service) are grasp the location of a person or thing based on a mobile network. Then, it is a concept that collectively the application systems and services to take advantage of it. In other words, it means a service provided by the location-based information to the user using various mobile devices such as mobile phone, PDA in the wired and the wireless mobile communication environment. As such LBS have emerged as a major key technology of basis infrastructure for location-based services with the development of wireless communication and Internet. Also ranging from individual, business, and public sector are utilized in a variety of forms. But, in case of service of the GPS and Cell-ID mode provided by the mobile phone in the domestic, GPS is impossible to grasp the position of underground or inside buildings. The case of Cell-ID method is result of the grasp the position is larger differences for accuracy requirements level of user. In order to compensate for this problem has emerged Seamless LBS [1]. Provided location information technology is essential to users that not free from crimes. Namely, In order to provide a user location lookup was unthinkable by separating the positionbased services such as GPS, LBS. In this paper, we investigated definition and technology for Seamless LBS that accuracy is higher than accuracy of GPS in mobile environment. And, we proposed a new location lookup framework that complements traditional location lookup techniques.

\section{RELATED RESEARCH}

\section{A. LBS(Loacation Based Service)}

The definition for the LBS is representing a little differently according each institution. OGC (Open GIS Consortium) is defined as all application software service that acts by the location information or connection and provide of location information. Also, the FCC (Federal Communication Commission) in the United States defines that receive service about geographical position, material or a known presence of mobile user. As such, each agency has defined variously. Generally follows the definition of the OGC.

LBS technology is the same context that developed of mobile communication technology. Namely, LBS technology is track the location of mobile devices in a variety of wired or wireless communication network. And it is collected and processed. Then, it is to service and to making a variety of content using that in the private and public sectors. Thus, location-based service means to create related applications that identify the location of the user using the location detection (check) techniques [3].

\section{B. Seamless LBS}

In case of service of the GPS and Cell-ID mode provided by the mobile phone in the domestic, GPS is impossible to grasp the position of underground or inside buildings. The case of Cell-ID method is result of the grasp the position is larger differences for accuracy requirements level of user. In order to compensate for this problem has emerged Seamless LBS.

Seamless LBS mean to LBS technology of the next generation that connected constantly location-based services of the center of the room and Small area.

In order to implement this, the indoor positioning technologies such as WLAN, Bluetooth is needed. Also to mobile terminal tracking requires a network and GPS outdoor positioning technology. Using the above two technologies integrate must be able to continuously provide position information. 


\section{Location-based Service Platform}

Location-Based Service Platform is a platform that provides most basic and critical function for LBS and it also provides a common component supporting a variety LBS. In this regard, many platforms have been developed like MPC, GISP, AS, and AP [2].

1) Mobile positioning center Mobile Positioning Center (MPC) is a server acting as elements for the location data retrieval, transmission, storage, and control in the network infrastructure. The Mobile Positioning Center is the entity that handles position information in cellular networks that use ANSI-41 for signalling (TR-45, 2002) [7, 8]. It uses a Position Determining Entity (PDE) to determine the position using a variety of technologies such as assisted GPS or observed time difference of arrival. MPC and MSC communicate with the emergency services network. The MPC also handles access restrictions to the position information.

2) Geographic information service platform Geographic Information Service Platform is a platform or content server giving contents as like POI, traffic information and electronic maps for LBS services. GISP uses spatial-temporal location as the key index variable for all other information. Just as a relational database containing text or numbers can relate many different tables using common key index variables, GISP can relate unrelated information by using location as the key index variable. The key is the location and/or extent in space-time [10].

3) Application server and service provider Application Server and Service Provider are acting like LBS application service provider or client server based on location information from Location-Based Service Platform or Geographic Information Service Platform. An application server can be either a software framework that provides a generalized approach to creating an application-server implementation, regard to what the application functions are, or the server portion of a specific implementation instance. In either case, the server's function is dedicated to the efficient execution of procedures (programs, routines, scripts) for supporting its applied applications [5, 6, 9].

\section{Framework}

Framework is a standardized system execution environment of application, the operating environment of the operator and the development environment of developer based on proven design and Re-use of source code.

High quality system development is possible, regardless of the ability by proceeding application development based on the standard framework. Also, there is saving effect of the institutional and cost of system construction. And it is possible to lower TCO and to reduce failures possible due to avoided of system complexity. It will be possible to secure the fundamental IT competitiveness [4].

\section{PROPSED FRAMEWORK}

The user threat to various crimes is increasing. So, to defend using traditional location provided technology is difficult. As described earlier, the development and research on the user location information is proceeding.

Services for the current position information are provided the GPS, Cell-ID by the way. So it is impossible to grasp a location inside buildings or underground. And the accuracy of the location information is not satisfactory. Indoor and outdoor location measurement using a Seamless LBS order to solve these problems is needed. If you follow the "checklist" your paper will conform to the requirements of the publisher and facilitate a problem-free publication process.

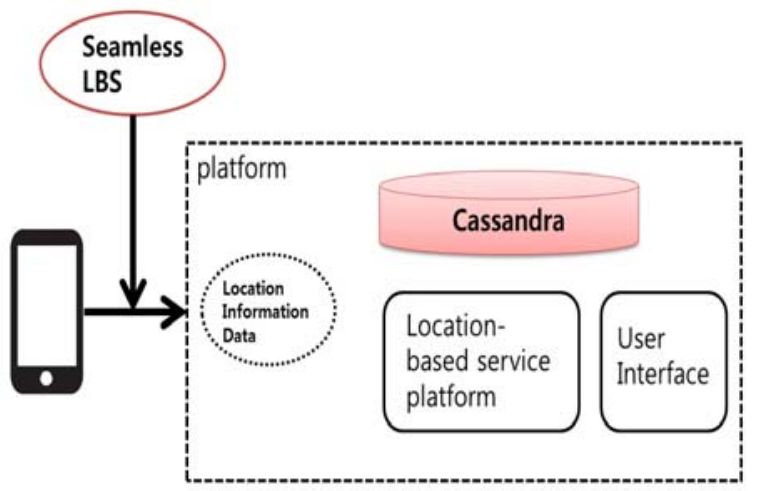

FIGURE I. PROPOSED FRAMEWORK

Proposed framework provides intelligent services by using the location information to be interoperable Different environments as well as a mobile environment. After that, it will be satisfy a variety of user requirements by connecting with the add-on and location-based services. Developed a framework is configured to interact with the user's needs and user position information that generating in a variety of situations. Also, the analyse using the Cassandra, one of the big data analysis techniques in order to the management of unstructured data, including location information. Cassandra is processing streaming data, one of kind complex event processing (CEP). It can be applied to stream data analysis quickly without additional infrastructure. Develop a unified interface environment based on this framework design. In order for service in a mobile environment must provide UI (User Interface) a high satisfaction to the user. If you build a service platform that includes Seamless LBS, it is expected to be used as a service technology to deliver and to retrieve user location information in mobile environments..

\section{CONCLUSION}

Location-based services have been studied and developed in a wide variety application area that has a close connection with our life. But, situation of development for technology or services for precise location information as yet is lacking. Therefore, the study should be continued about Seamless LBS in order to grasp when the user's location information anywhere. So, shall provide a high accuracy about position. Also, it may provide various associated services to meet the user requirements consist the data analysis on the basis of this 
position information. And it is determined that a little higher location lookup service is to be made by providing a user interface to high satisfaction.

Problem with respect to various position information technologies in many fields should analyse. Also, if come true about various service and system research and development, is helping provide highly accurate in position information from a variety of IT environments.

\section{ACKNOWLEDGMENT}

This work (Grants No.C0199617) was supported by business for Cooperative R\&D between Industry, Academy, and Research Institute funded Korea Small and Medium Business Administration in 2014.

\section{REFERENCES}

[1] Jindam Mok, Pyungdong Cho, The Review on LBS Technology and Future Trends, Korea Information and Communications Society, 2004.
[2] H. Choi, LBS, Location-Based Services trend Computer Software Research, Korea Electronics and Telecommunications Research Institute, No.86, 2003.

[3] S.H Park, Location-Based Services (LBS) technology and market trends, Technical note, Surveying \& Mapping Magazine, 3, 2011.

[4] S.H Yoon, S.Y Cho, J.W Choi, A study on Frameworks for Platform of 3D Indoor Spatial Information, Korea Interior Design Journal, 14(1), pp. 210-213, 2012.

[5] Y. Zhao, Standardization of mobile phone positioning for $3 G$ systems, IEEE Communication. Mag, 40(7), pp. 108-116, 2002.

[6] Y. Jaejun et al, Interior space, a new chapter in the fusion attention, Electronics and Telecommunications Research Institute, No. 1571, pp. 14-16, 2011.

[7] Y. Cho et al, WARP-P: Wireless signal Acquisition with Reference Point by using simplified PDF - sys-tem concept and performance assessment, Proc. The ION 2013 Pacific PNT Meeting, Apr, 2013.

[8] D. Tipper, Network architecture and protocols for mobile positioning in cellular wireless systems

[9] Application server, Wikipedia.org/wiki/Application server

[10] Geographic information platform, Wikipedia.org/wiki/Geographic information platform 